Research Article

\title{
Game-Theoretic Models of Green Products in a Two-Echelon Dual-Channel Supply Chain under Government Subsidies
}

\author{
Zhenkai Lou $\mathbb{D}^{\mathrm{D}},{ }^{1}$ Xuming Lou $\mathbb{D D}^{2}{ }^{2}$ and Xiaozhen Dai ${ }^{3}{ }^{3}$ \\ ${ }^{1}$ School of Management and Economics, Beijing Institute of Technology, Beijing, China \\ ${ }^{2}$ School of Economics and Management, Xi'an University of Posts and Telecommunications, Xi'an, Shaanxi, China \\ ${ }^{3}$ School of Management, Wenzhou Business College, Wenzhou, Zhejiang, China
}

Correspondence should be addressed to Xiaozhen Dai; 18810788027@139.com

Received 24 February 2020; Revised 20 April 2020; Accepted 21 April 2020; Published 28 May 2020

Academic Editor: Przemyslaw Ignaciuk

Copyright ( $\odot 2020$ Zhenkai Lou et al. This is an open access article distributed under the Creative Commons Attribution License, which permits unrestricted use, distribution, and reproduction in any medium, provided the original work is properly cited.

This paper deals with issues concerning green subsidies of government and optimal decisions of a manufacture and dual-channel retailers in a two-echelon dual-channel supply chain. Both a decentralized supply chain and a centralized supply chain are considered. Sufficient and necessary conditions for guaranteeing that the two supply chains run normally under government subsidies are proposed. For the decentralized supply chain, a three-layer model is constructed according to different priorities of the four participants. Both Bertrand game and Stackelberg game are involved. For the centralized supply chain, a two-layer model is given. Decision models of the government under a financial budget are developed for maximizing the green degree of each case. It is shown that the green degree of the product of the centralized supply chain is always higher than that of the decentralized supply chain. Meanwhile, the total profit of the centralized supply is also higher. Finally, a numerical illustration is presented to visualize the discussed models and make some supplements.

\section{Introduction}

Environmental consciousness has become increasingly important in everyday life and business practice [1]. Raising the consumer's demand for environmentally friendlier products with less harmful environmental effects is an important measure to protect the environment and save energies [2]. However, both manufacturers and consumers prefer old-fashioned production because of its low cost [3]. Hence, governments introduce environmentally friendly regulations so as to enhance the positivity of both manufacturers and consumers for green products [4].

In recent years, because of the high practicability, production and pricing strategies on green products attract much attention and a growing body of the literature focuses on production and pricing strategies of green products in green supply chains. Swami and Shah [1] addressed some interesting questions such as extent of effort in greening of operations by the manufacturer or retailer, level of cooperation between the two parties, and how to coordinate their operations in a supply chain. Zhang et al. [5] investigated energy efficiency level and pricing policies in a single manufacturer-retailer setup, in which the production cost is affected by both cost learning and operational inefficiency effects. With a game-theoretic approach, Zhu and He [6] investigated the green product design issues of how supply chains' decisions on the greenness of products are affected by supply chain structures. Song and Gao [7] focused on different revenue-sharing contracts of the whole green supply chain and compared the results of the common centralized model and the decentralized decision model.

In practice, government subsidies always play a crucial role in pricing and the choice of performance levels of green products [8]. For example, in China, the energy-saving subsidies have significantly promoted the production and sales of energy-saving electric appliances and new energy automobiles. Some literature studies focus on this aspect when dealing with production and pricing issues. Zhang et al. [9] analyzed subsidy policies of the government to provide greater incentives for industry manufactures. Yu 
et al. [10] discussed the decision-making issue faced by manufacturers to determine the levels of green products to produce and production quantities at each green level. Hafezalkotob [11] compared six different policies in priceenergy saving and used these policies to study the two structures of centralized and decentralized green supply chains. Safarzadeh and Rasti-Barzoki [12] studied a sustainable supply chain which involves an energy-saving manufacturer, inefficient manufacturer, and energy supplier and constructed a multistage game model under government policies of tax deduction and subsidy scheme. Nielsen et al. $[13,14]$ analyzed optimal decision strategies under different subsidized objects and proposed twelve analytical models by considering the impact of different game structures.

With the development of internet techniques, more and more people prefer to purchase through e-commerce platform. Owing to this, many manufacturers redesign their traditional channel structures by engaging in direct sales [15]. The selling channel brought by the internet endows the manufacturers with more opportunities for cost savings, revenue growth, and expansion to new market segments [16]. Owing to the above reasons, some literature studies considered dual channels while discussing production and pricing issues of green products. Li et al. [17] considered a dual-channel green supply chain and discussed the pricing and greening policies for both centralized and decentralized situations by adopting the Stackelberg game. Javadi et al. [18] discussed government intervention policies of energy-saving, revenue-seeking, social welfare, and sustainable development in a dual-channel supply chain.

Our study is motivated by the above literature. The basic setting of this study is that a manufacturer produces green products under government subsidies and dual-channel retailers sell them to consumers. Similar to Liu and Yi [19], the manufacturer determines the green degree level of the product and the government offers subsidies to him. Stimulated by Saha et al. [20], both centralized and decentralized cases are studied. For the decentralized case, a three-layer decision model is developed according to the decision order of each decision maker. Both Bertrand game and Stackelberg game are conducted to obtain optimal decision strategies. For the centralized case, the manufacturer and the two retailers jointly determine the green degree and sales prices. In this scenario, we pay no attention to the wholesale price because it does not make any impact on optimal decision. The differences on the green degree subsidies and the total profit between the two cases are compared. The sensitivity of each supply chain with regard to the coefficient of the extra production cost is shown by a curve graph.

Our research yields some theoretical and practical results. First, the existence and uniqueness of each solution of the two supply chains is shown. Second, a sufficient condition for guaranteeing that each manufacturer of the two supply chains has a unique and reasonable solution under government subsidies is shown. Third, at the point of view of the government, an approach is developed to make policies of green-product subsidies under a finite financial budget.
Finally, we demonstrate that the green degree of the product of the centralized supply chain is always higher than the one of the decentralized supply chain.

The remainder of this paper is organized as follows: necessary notations and assumptions are given in Section 2; Section 3 conducts the decentralized decision model involving a Bertrand game and a Stackelberg game, aiming to discuss the theoretical and practical meanings of the model and acquire optimal solutions for all the participants; in Section 4, a centralized supply chain model is presented; a numerical illustration is shown in Section 5 to make some supplements; Section 6 summarizes the whole paper and shows the future research prospects.

\section{Notations and Assumptions}

This paper considers green product selling issues involving a monopolistic manufacturer, an online retailer, and a traditional entity retailer. In addition, the government makes subsidy policies under a finite financial budget to maximize the green degree of products. Given the above, the considered problem can be formulated as a three-layer decision model.

Necessary notations used in the following discussion are given as follows:

$a$ : the potential product demands of the market for the product.

$\varphi$ : the coefficient of the extra production cost related to the green degree, $\varphi>0$.

$\tau$ : the green degree of the product, $\tau>0$.

$\lambda$ : the purchasing preference proportion for the traditional retailer; thus, $1-\lambda$ measures the purchasing preference proportion for the platform channel. Clearly, $0<\lambda<1$.

$\delta$ : the marginal demand with respect to the sales price, $\delta>0$.

$\theta$ : the demand shift between the two channels with respect to the price, $\theta>0$.

$\beta$ : the marginal demand with respect to the green degree, $\beta>0$.

$q_{b}$ : the wholesale price given by the manufacturer for the physical store.

$q_{e}$ : the wholesale price given by the manufacturer for the online store.

$p_{b}$ : the sales price determined by the physical store. $p_{e}$ : the sales price determined by the online store.

$D_{b}$ : the sales (demand) quantity of the physical store during the given sales period, $D_{b}=\lambda a-\delta p_{b}+\theta p_{e}+\beta_{\tau}$.

$D_{e}$ : the sales (demand) quantity of the online store during the given sales period, $D_{e}=(1-\lambda)$ $\mathrm{a}-\delta p_{e}+\theta p_{b}+\beta_{\tau}$.

$g$ : the financial budget of the government to motivate green production for the manufacturer, $g>0$.

$k$ : the per-unit green degree subsidy given by the government, $k>0$. 
$M$ : the total revenue of the manufacturer.

$R_{b}$ : the total revenue of the physical store.

$R_{e}$ : the total revenue of the online store.

Some explanations are given for the above formulae. In almost all the related literature, the subsidy threshold value of the green degree is always a constant. Thus, the green degree in this paper can be regarded as the value of the green degree determined by the manufacturer minus the subsidy threshold value.

Without loss of generality, we assume that the physical store attracts more purchasing preferences when the two sales prices are the same, i.e., $\lambda>1 / 2$. It is acknowledged that one channel's own price effect is greater than the cross-price effect, namely, $\delta>\theta$. Furthermore, similar to the assumptions of Li et al. [17] and Hafezalkotob [21] with regard to the relationships of coefficients of the demand function, we assume that $\delta>\theta>\beta$. Generally speaking, the market demand is more sensitive to prices than to the green degree, which is reasonable and is usually the case [4].

Following Ghosh and Shah [22] and Li et al. [17], the extra cost for the manufacturer to produce the green product is denoted by

$$
\left(\frac{1}{2}\right) \varphi \tau^{2}
$$

For the sake of the following discussion, some premises are given as follows:

(1) This paper considers a monopolistic manufacturer who produces irreplaceable products; i.e., no other substitutable product exists.

(2) All of them, the manufacturer, two retailers, and the government, are able to acquire complete information about the market and the revenue function.

(3) The production capacity of the manufacturer is assumed to be sufficient to meet the market demand.

(4) Both the physical store retailer and the e-commerce retailer can only price the product for one time throughout the whole sales period. Namely, once the price of each channel is determined at the beginning of the period, it cannot be changed.

(5) Different demand rates seriously affect the stockholding cost, yet this paper only considers demand quantity instead of demand rate. Hence, we assume that the stock-holding cost is a constant so that it does not affect the decision of each participant.

\section{Three-Layer Model}

In this section, we construct a decentralized decisionmaking two-echelon green supply chain model which involves a Stackelberg game between the manufacturer and the two retailers and a Bertrand game between the two retailers. In addition to the supply chain, the government offers subsidies for the green degree of products under a finite financial budget. Hence, the discussed issue is a three-layer problem which involves four participants.

The contrary recurrence approach is adopted to handle this model. Before analyzing solutions, we present the objective of each participant.

On the premise that the financial budget for motivating green production is $g$, the government aims to determine the green degree subsidy $k$ to maximize the green degree level $\tau$.

The objective function of the monopolistic manufacturer is given as follows:

$$
M=q_{b} D_{b}+q_{e} D_{e}-\left(\frac{1}{2}\right) \varphi \tau^{2}+k \tau,
$$

where $k$ is uncertain by now. Decision variables of the manufacturer are $q_{b}, q_{e}$, and $\tau$.

The objective function of the brick and mortar store with $p_{b}$ as its variable is

$$
R_{b}=\left(p_{b}-q_{b}\right)\left(\lambda a-\delta p_{b}+\theta p_{e}+\beta \tau\right) .
$$

Similarly, the objective function of the e-commerce store with $p_{e}$ as its variable is

$$
R_{e}=\left(p_{e}-q_{e}\right)\left[(1-\lambda) a-\delta p_{e}+\theta p_{b}+\beta \tau\right] .
$$

Objective functions (2), (3), and (4) compose a Stackelberg game, in which the manufacturer is the leader and the two retailers are followers. Besides, the two retailers, the brick and mortar store, and the e-commerce store form a Bertrand game. First, we deal with the Bertrand game on the premise that $q_{b}, q_{e}$, and $\tau$ are fixed.

By differentiating $R_{b}$, we obtain the following equation:

$$
\frac{\partial R_{b}}{\partial p_{b}}=-2 \delta p_{b}+\theta p_{e}+\lambda a+\beta \tau+\delta q_{b}=0 .
$$

Examining the second-order derivative of $R_{b}$, we have

$$
\frac{\partial^{2} R_{b}}{\partial p_{b}^{2}}=-2 \delta<0
$$

which means that the solution of equation (5) is the unique solution of $\max R_{b}$.

Similarly, the corresponding equation of $R_{e}$ is

$$
\frac{\partial R_{e}}{\partial p_{e}}=-2 \delta p_{e}+\theta p_{b}+(1-\lambda) a+\beta \tau+\delta q_{e}=0 .
$$

Examining the second-order derivative of $R_{e}$, we have

$$
\frac{\partial^{2} R_{e}}{\partial p_{e}^{2}}=-2 \delta<0
$$

Thus, the solution of equation (7) is the unique solution of $\max R_{e}$.

We denote by $p_{b}^{*}$ and $p_{e}^{*}$ the solution of equations (5) and (7). Handling equations (5) and (7) by the elimination 
method, the unique equilibrium solution of the Bertrand game is acquired as follows:

$$
\left\{\begin{array}{l}
p_{b}^{*}=\frac{2 \delta \lambda a+\theta(1-\lambda) a+2 \delta^{2} q_{b}+\delta \theta q_{e}+(2 \delta+\theta) \beta \tau}{4 \delta^{2}-\theta^{2}}, \\
p_{e}^{*}=\frac{2 \delta(1-\lambda) a+\theta \lambda a+\delta \theta q_{b}+2 \delta^{2} q_{e}+(2 \delta+\theta) \beta \tau}{4 \delta^{2}-\theta^{2}} .
\end{array}\right.
$$

By solution (9), we focus on dealing with the Stackelberg game. Objective function (2) is transformed to

$$
\begin{aligned}
M= & \frac{1}{4 \delta^{2}-\theta^{2}}\left[-\left(2 \delta^{3}-\delta \theta^{2}\right) q_{b}^{2}+2 \delta^{2} \lambda a q_{b}\right. \\
& \left.+\delta \theta(1-\lambda) a q_{b}+\left(2 \delta^{2}+\delta \theta\right) \beta \tau q_{b}\right] \\
& +\frac{1}{4 \delta^{2}-\theta^{2}}\left[-\left(2 \delta^{3}-\delta \theta^{2}\right) q_{e}^{2}+2 \delta^{2}(1-\lambda) a q_{e}\right. \\
& \left.+\delta \theta \lambda a q_{e}+\left(2 \delta^{2}+\delta \theta\right) \beta \tau q_{e}\right] \\
& +\frac{2 \delta^{2} \theta}{4 \delta^{2}-\theta^{2}} q_{b} q_{e}-\frac{1}{2} \varphi \tau^{2}+k \tau .
\end{aligned}
$$

Proof. Handling the above determinant by the reducedorder method, we have

Before handling the solution of $\max M$, we first present the following proposition:

Lemma 1. Under $\delta>\theta>0$, the following inequality

$$
\operatorname{Det}=\left|\begin{array}{ccc}
-\frac{4 \delta^{3}-2 \delta \theta^{2}}{4 \delta^{2}-\theta^{2}} & \frac{2 \delta^{2} \theta}{4 \delta^{2}-\theta^{2}} & \frac{\left(2 \delta^{2}+\delta \theta\right) \beta}{4 \delta^{2}-\theta^{2}} \\
\frac{2 \delta^{2} \theta}{4 \delta^{2}-\theta^{2}} & -\frac{4 \delta^{3}-2 \delta \theta^{2}}{4 \delta^{2}-\theta^{2}} & \frac{\left(2 \delta^{2}+\delta \theta\right) \beta}{4 \delta^{2}-\theta^{2}} \\
\frac{\left(2 \delta^{2}+\delta \theta\right) \beta}{4 \delta^{2}-\theta^{2}} & \frac{\left(2 \delta^{2}+\delta \theta\right) \beta}{4 \delta^{2}-\theta^{2}} & -\varphi
\end{array}\right|<0,
$$

is equivalent to

$$
\left(4 \delta^{2}-2 \theta^{2}\right)^{2} \varphi-4 \delta^{2} \theta^{2} \varphi-4(\delta+\theta)\left(2 \delta^{2}+\delta \theta\right) \beta^{2}>0 .
$$

$$
\begin{aligned}
\left|\begin{array}{ccc}
-\frac{4 \delta^{3}-2 \delta \theta^{2}}{4 \delta^{2}-\theta^{2}} & \frac{2 \delta^{2} \theta}{4 \delta^{2}-\theta^{2}} & \frac{\left(2 \delta^{2}+\delta \theta\right) \beta}{4 \delta^{2}-\theta^{2}} \\
\frac{2 \delta^{2} \theta}{4 \delta^{2}-\theta^{2}} & -\frac{4 \delta^{3}-2 \delta \theta^{2}}{4 \delta^{2}-\theta^{2}} & \frac{\left(2 \delta^{2}+\delta \theta\right) \beta}{4 \delta^{2}-\theta^{2}} \\
\frac{\left(2 \delta^{2}+\delta \theta\right) \beta}{4 \delta^{2}-\theta^{2}} \frac{\left(2 \delta^{2}+\delta \theta\right) \beta}{4 \delta^{2}-\theta^{2}} & -\varphi
\end{array}\right|=-\frac{4 \delta^{3}-2 \delta \theta^{2}}{4 \delta^{2}-\theta^{2}}\left|\begin{array}{cc}
-\frac{4 \delta^{3}-2 \delta \theta^{2}}{4 \delta^{2}-\theta^{2}} & \frac{\left(2 \delta^{2}+\delta \theta\right) \beta}{4 \delta^{2}-\theta^{2}} \\
\frac{\left(2 \delta^{2}+\delta \theta\right) \beta}{4 \delta^{2}-\theta^{2}} & -\varphi
\end{array}\right| \\
-\frac{2 \delta^{2} \theta}{4 \delta^{2}-\theta^{2}}\left|\begin{array}{cc}
\frac{2 \delta^{2} \theta}{4 \delta^{2}-\theta^{2}} & \frac{\left(2 \delta^{2}+\delta \theta\right) \beta}{4 \delta^{2}-\theta^{2}} \\
\frac{\left(2 \delta^{2}+\delta \theta\right) \beta}{4 \delta^{2}-\theta^{2}} & -\varphi
\end{array}\right|+\frac{\left(2 \delta^{2}+\delta \theta\right) \beta}{4 \delta^{2}-\theta^{2}}\left|\begin{array}{cc}
\frac{2 \delta^{2} \theta}{4 \delta^{2}-\theta^{2}} & -\frac{4 \delta^{3}-2 \delta \theta^{2}}{4 \delta^{2}-\theta^{2}} \\
\frac{\left(2 \delta^{2}+\delta \theta\right) \beta}{4 \delta^{2}-\theta^{2}} & \frac{\left(2 \delta^{2}+\delta \theta\right) \beta}{4 \delta^{2}-\theta^{2}}
\end{array}\right| \\
= \\
=\frac{\left[-\left(4 \delta^{2}-\theta^{2}\right)\left(4 \delta^{3}-2 \delta \theta^{2}\right)^{2} \varphi+\left(8 \delta^{3}+4 \delta^{2} \theta-4 \delta \theta^{2}\right)\left(2 \delta^{2}+\delta \theta\right)^{2} \beta^{2}+4\left(4 \delta^{2}-\theta^{2}\right) \delta^{4} \theta^{2} \varphi\right.}{\left(4 \delta^{2}-\theta^{2}\right)^{3}}
\end{aligned}
$$

Because $\delta>\theta>0$, (11) is equivalent to

$$
\begin{gathered}
\left(4 \delta^{2}-\theta^{2}\right)\left(4 \delta^{2}-2 \theta^{2}\right)^{2} \delta^{2} \varphi-\left(8 \delta^{3}+4 \delta^{2} \theta-4 \delta \theta^{2}\right)\left(2 \delta^{2}+\delta \theta\right)^{2} \beta^{2}-4\left(4 \delta^{2}-\theta^{2}\right) \delta^{4} \theta^{2} \varphi>0 \\
\left(4 \delta^{2}-\theta^{2}\right) \delta^{2}>0
\end{gathered}
$$


we find that (11) is also equivalent to

$$
\left(4 \delta^{2}-2 \theta^{2}\right)^{2} \varphi-\frac{\left(8 \delta^{3}+4 \delta^{2} \theta-4 \delta \theta^{2}\right)\left(2 \delta^{2}+\delta \theta\right)}{4(\delta+\theta)\left(4 \delta^{2}-\theta^{2}\right) \delta^{2}} 4(\delta+\theta)\left(2 \delta^{2}+\delta \theta\right) \beta^{2}-4 \delta^{2} \theta^{2} \varphi>0 .
$$

Because

$$
\left(8 \delta^{3}+4 \delta^{2} \theta-4 \delta \theta^{2}\right)\left(2 \delta^{2}+\delta \theta\right)=4 \delta^{2}(\delta+\theta)(2 \delta-\theta)(2 \delta+\theta)=4(\delta+\theta)\left(4 \delta^{2}-\theta^{2}\right) \delta^{2},
$$

(16) is equal to (12).

According to the transitivity of the equivalence relation, (11) is equivalent to (12).

For the solution of $\max M$, we show the following conclusion:
Theorem 1. (11) is a sufficient condition for guaranteeing that $\max M$ has a unique and reasonable solution.

Proof. All first-order partial derivatives of $M$ are

$$
\left\{\begin{array}{l}
\frac{\partial M}{\partial q_{b}}=-\frac{4 \delta^{3}-2 \delta \theta^{2}}{4 \delta^{2}-\theta^{2}} q_{b}+\frac{2 \delta^{2} \theta}{4 \delta^{2}-\theta^{2}} q_{e}+\frac{2 \delta^{2} \lambda a+\delta \theta(1-\lambda) a+\left(2 \delta^{2}+\delta \theta\right) \beta \tau}{4 \delta^{2}-\theta^{2}} \\
\frac{\partial M}{\partial q_{e}}=-\frac{4 \delta^{3}-2 \delta \theta^{2}}{4 \delta^{2}-\theta^{2}} q_{e}+\frac{2 \delta^{2} \theta}{4 \delta^{2}-\theta^{2}} q_{b}+\frac{2 \delta^{2}(1-\lambda) a+\delta \theta \lambda a+\left(2 \delta^{2}+\delta \theta\right) \beta \tau}{4 \delta^{2}-\theta^{2}} \\
\frac{\partial M}{\partial \tau}=\frac{\left(2 \delta^{2}+\delta \theta\right) \beta}{4 \delta^{2}-\theta^{2}} q_{b}+\frac{\left(2 \delta^{2}+\delta \theta\right) \beta}{4 \delta^{2}-\theta^{2}} q_{e}-\varphi \tau+k
\end{array}\right.
$$

By deriving second-order derivatives of $M$, we have the corresponding Hessian matrix as follows:

$$
H=\left[\begin{array}{ccc}
\frac{\partial^{2} M}{\partial q_{b}^{2}} & \frac{\partial^{2} M}{\partial q_{b} \partial q_{e}} & \frac{\partial^{2} M}{\partial q_{b} \partial \tau} \\
\frac{\partial^{2} M}{\partial q_{e} \partial q_{b}} & \frac{\partial^{2} M}{\partial q_{e}^{2}} & \frac{\partial^{2} M}{\partial q_{e} \partial \tau} \\
\frac{\partial^{2} M}{\partial \tau \partial q_{b}} & \frac{\partial^{2} M}{\partial \tau \partial q_{e}} & \frac{\partial^{2} M}{\partial \tau^{2}}
\end{array}\right]=\left[\begin{array}{ccc}
-\frac{4 \delta^{3}-2 \delta \theta^{2}}{4 \delta^{2}-\theta^{2}} & \frac{2 \delta^{2} \theta}{4 \delta^{2}-\theta^{2}} & \frac{\left(2 \delta^{2}+\delta \theta\right) \beta}{4 \delta^{2}-\theta^{2}} \\
\frac{2 \delta^{2} \theta}{4 \delta^{2}-\theta^{2}} & -\frac{4 \delta^{3}-2 \delta \theta^{2}}{4 \delta^{2}-\theta^{2}} & \frac{\left(2 \delta^{2}+\delta \theta\right) \beta}{4 \delta^{2}-\theta^{2}} \\
\frac{\left(2 \delta^{2}+\delta \theta\right) \beta}{4 \delta^{2}-\theta^{2}} & \frac{\left(2 \delta^{2}+\delta \theta\right) \beta}{4 \delta^{2}-\theta^{2}} & -\varphi
\end{array}\right] .
$$

Because $\delta>\theta$, by examining each order determinant of $H$, we find that

$$
\begin{gathered}
-\frac{4 \delta^{3}-2 \delta \theta^{2}}{4 \delta^{2}-\theta^{2}}<0, \\
\left|\begin{array}{cc}
-\frac{4 \delta^{3}-2 \delta \theta^{2}}{4 \delta^{2}-\theta^{2}} & \frac{2 \delta^{2} \theta}{4 \delta^{2}-\theta^{2}} \\
\frac{2 \delta^{2} \theta}{4 \delta^{2}-\theta^{2}} & -\frac{4 \delta^{3}-2 \delta \theta^{2}}{4 \delta^{2}-\theta^{2}}
\end{array}\right|=\frac{\left(4 \delta^{3}-2 \delta \theta^{2}\right)^{2}}{\left(4 \delta^{2}-\theta^{2}\right)^{2}}-\frac{4 \delta^{4} \theta^{2}}{\left(4 \delta^{2}-\theta^{2}\right)^{2}}>\frac{4 \delta^{6}-4 \delta^{4} \theta^{2}}{\left(4 \delta^{2}-\theta^{2}\right)^{2}}>0 .
\end{gathered}
$$


Hence, inequality (11) guarantees that $H$ is negative definite. Consider the following equation set derived from (18):

$$
\left\{\begin{array}{l}
-\frac{4 \delta^{3}-2 \delta \theta^{2}}{4 \delta^{2}-\theta^{2}} q_{b}+\frac{2 \delta^{2} \theta}{4 \delta^{2}-\theta^{2}} q_{e}+\frac{2 \delta^{2} \lambda a+\delta \theta(1-\lambda) a+\left(2 \delta^{2}+\delta \theta\right) \beta \tau}{4 \delta^{2}-\theta^{2}}=0 \\
-\frac{4 \delta^{3}-2 \delta \theta^{2}}{4 \delta^{2}-\theta^{2}} q_{e}+\frac{2 \delta^{2} \theta}{4 \delta^{2}-\theta^{2}} q_{b}+\frac{2 \delta^{2}(1-\lambda) a+\delta \theta \lambda a+\left(2 \delta^{2}+\delta \theta\right) \beta \tau}{4 \delta^{2}-\theta^{2}}=0 \\
\frac{\left(2 \delta^{2}+\delta \theta\right) \beta}{4 \delta^{2}-\theta^{2}} q_{b}+\frac{\left(2 \delta^{2}+\delta \theta\right) \beta}{4 \delta^{2}-\theta^{2}} q_{e}-\varphi \tau+k=0 .
\end{array}\right.
$$

By equation (22), we obtain expressions of $q_{b}$ and $q_{e}$ as follows:

$$
\left\{\begin{array}{l}
q_{b}=\frac{8 \delta^{3} \lambda a+8 \delta^{2} \theta(1-\lambda) a-2 \delta \theta^{2} \lambda a-2 \theta^{3}(1-\lambda) a+\left(8 \delta^{3}+8 \delta^{2} \theta-2 \delta \theta^{2}-2 \theta^{3}\right) \beta \tau}{\left(4 \delta^{2}-2 \theta^{2}\right)^{2}-4 \delta^{2} \theta^{2}} \\
q_{e}=\frac{8 \delta^{3}(1-\lambda) a+8 \delta^{2} \theta \lambda a-2 \delta \theta^{2}(1-\lambda) a-2 \theta^{3} \lambda a+\left(8 \delta^{3}+8 \delta^{2} \theta-2 \delta \theta^{2}-2 \theta^{3}\right) \beta \tau}{\left(4 \delta^{2}-2 \theta^{2}\right)^{2}-4 \delta^{2} \theta^{2}}
\end{array}\right.
$$

Then, equation (22) is transformed to

$$
\frac{\beta\left(2 \delta^{2}+\delta \theta\right)[2 \delta a+2 \theta a+4(\delta+\theta) \beta \tau]}{\left(4 \delta^{2}-2 \theta^{2}\right)^{2}-4 \delta^{2} \theta^{2}}-\varphi \tau+k=0,
$$

by which we have

$$
\tau^{*}=\frac{2 \beta\left(2 \delta^{2}+\delta \theta\right)(\delta a+\theta a)+\left(4 \delta^{2}-2 \theta^{2}\right)^{2} k-4 \delta^{2} \theta^{2} k}{\left[\left(4 \delta^{2}-2 \theta^{2}\right)^{2} \varphi-4 \delta^{2} \theta^{2} \varphi-4(\delta+\theta)\left(2 \delta^{2}+\delta \theta\right) \beta^{2}\right]}
$$

where $\tau^{*}$ is the solution of (24). (23):

Then, $q_{b}^{*}$ and $q_{e}^{*}$ are acquired by substituting (25) into

$$
\left\{\begin{array}{l}
q_{b}^{*}=\frac{8 \delta^{3} \lambda a+8 \delta^{2} \theta(1-\lambda) a-2 \delta \theta^{2} \lambda a-2 \theta^{3}(1-\lambda) a}{\left(4 \delta^{2}-2 \theta^{2}\right)^{2}-4 \delta^{2} \theta^{2}}+C, \\
q_{e}^{*}=\frac{8 \delta^{3}(1-\lambda) a+8 \delta^{2} \theta \lambda a-2 \delta \theta^{2}(1-\lambda) a-2 \theta^{3} \lambda a}{\left(4 \delta^{2}-2 \theta^{2}\right)^{2}-4 \delta^{2} \theta^{2}}+C,
\end{array}\right.
$$

where $C$ is a constant given as follows:

$$
C=\frac{\left(8 \delta^{3}+8 \delta^{2} \theta-2 \delta \theta^{2}-2 \theta^{3}\right)\left[2 \beta\left(2 \delta^{2}+\delta \theta\right)(\delta a+\theta a)+\left(4 \delta^{2}-2 \theta^{2}\right)^{2} k-4 \delta^{2} \theta^{2} k\right] \beta}{\left[\left(4 \delta^{2}-2 \theta^{2}\right)^{2}-4 \delta^{2} \theta^{2}\right]\left[\left(4 \delta^{2}-2 \theta^{2}\right)^{2} \varphi-4 \delta^{2} \theta^{2} \varphi-4(\delta+\theta)\left(2 \delta^{2}+\delta \theta\right) \beta^{2}\right]}
$$

By Lemma 1, we know that (11) is equivalent to (12). Besides, by $\delta>\theta$, we have

$$
\left(4 \delta^{2}-2 \theta^{2}\right)^{2} k-4 \delta^{2} \theta^{2} k>4 \delta^{4} k-4 \delta^{2} \theta^{2} k>0 .
$$

Hence, $\tau^{*}>0$ under (11), i.e., (11) is a sufficient condition for guaranteeing that $\max M$ has a unique and reasonable solution.
After the manufacturer declares $q_{b}^{*}, q_{e}^{*}$, and $\tau^{*}$, values of $p_{b}^{*}$ and $p_{e}^{*}$ are then obtained simultaneously by substituting (25) and (26) into (9).

We briefly analyze the reason why all the coefficients should meet (11) (or (12)) so as to guarantee that $\max M$ has a unique and reasonable solution.

If (11) (or (12)) does not hold, the unique extreme point of $M$ given by (25) and (26) is not the solution of $\max M$. By (23), 
$M$ is transformed to a one-variable function of $\tau$, which we denote by $M(\tau)$. According to the properties of differentiable functions, $M(\tau)$ must be a monotonic function on the domain of definition $(0,+\infty)$, which suggests that the green degree of the product should be zero or infinity (or a given upper limit) when maximizing the total revenue of the manufacturer. Usually, it is impractical for a green-product supply chain.

Given the above, (11) is also a necessary condition for guaranteeing that $\max M$ has a unique and reasonable solution.

Furthermore, we also have the following conclusion.

Proposition 1. A two-echelon dual-channel supply chain under government subsidies runs normally, if and only if all the parameters meet (11) (or (12)).

By this result, the government is able to make decisions of whether green-production subsidies should be offered to the manufacturer or not by analyzing all the parameters.

Next, the relation between the coefficient of the extra production cost and other parameters is examined.

By transforming (12), we have

$$
\varphi>\frac{4(\delta+\theta)\left(2 \delta^{2}+\delta \theta\right) \beta^{2}}{\left(4 \delta^{2}-2 \theta^{2}\right)^{2}-4 \delta^{2} \theta^{2}}
$$

which implies that the coefficient of the extra production cost is often higher than a certain value. Otherwise, the manufacturer will enhance the green degree to the upper limit under a coefficient of the extra production cost that does not meet (29). Hence, (29) always holds in practical cases.

Finally, we consider the per-unit green degree subsidy of the government. Regarding $\tau^{*}$ as a function of $k: \tau^{*}(k)$, the first-order derivative of $\tau^{*}(k)$ is obtained as follows:

$$
\frac{\partial \tau^{*}}{\partial k}=\frac{\left(4 \delta^{2}-2 \theta^{2}\right)^{2}-4 \delta^{2} \theta^{2}}{\left[\left(4 \delta^{2}-2 \theta^{2}\right)^{2} \varphi-4 \delta^{2} \theta^{2} \varphi-4(\delta+\theta)\left(2 \delta^{2}+\delta \theta\right) \beta^{2}\right]}>0 .
$$

which means that $\tau^{*}$ is strictly monotonically increasing with the increase of $k$.

Under a financial budget $g$, we consider the following model:

$$
\begin{array}{ll}
\max & k \\
\text { s.t. } & \frac{2 \beta\left(2 \delta^{2}+\delta \theta\right)(\delta a+\theta a) k+\left[\left(4 \delta^{2}-2 \theta^{2}\right)^{2}-4 \delta^{2} \theta^{2}\right] k^{2}}{\left[\left(4 \delta^{2}-2 \theta^{2}\right)^{2} \varphi-4 \delta^{2} \theta^{2} \varphi-4(\delta+\theta)\left(2 \delta^{2}+\delta \theta\right) \beta^{2}\right]} \leq g .
\end{array}
$$

In practice, the quadratic formula is adopted to acquire the maximum value of $k$ with regard to the inequality in model (31).

\section{Two-Layer Model}

This section deals with a two-layer decision model in which the manufacturer and the two retailers jointly make decisions. The government declares the per-unit green degree subsidy first, and then, the two-echelon supply chain determines the green degree and the sales prices. In practice, this case can be regarded that a monopolistic manufacturer possesses two channels to sell products, just like Amrouche and Yan [23] considered.

By the given notations, the profit function $S$ of a centralized supply chain with dual-sales channels is obtained as follows:

$$
\begin{aligned}
S= & \left(\lambda a-\delta p_{b}+\theta p_{e}+\beta \tau\right) p_{b}+\left[(1-\lambda) a-\delta p_{e}+\theta p_{b}+\beta \tau\right] p_{e} \\
& -\frac{1}{2} \varphi \tau^{2}+k \tau .
\end{aligned}
$$

Similarly, before handling the solution of $\max S$, we present the following proposition.

Lemma 2. Under $\delta>\theta>0$, the following inequality

$$
\operatorname{Det}=\left|\begin{array}{ccc}
-2 \delta & 2 \theta & \beta \\
2 \theta & -2 \delta & \beta \\
\beta & \beta & -\varphi
\end{array}\right|<0,
$$

is equivalent to

$$
2(\delta-\theta) \varphi-2 \beta^{2}>0 .
$$

Proof. Handling the above determinant by the reducedorder method, we have

$$
\begin{aligned}
\left|\begin{array}{ccc}
-2 \delta & 2 \theta & \beta \\
2 \theta & -2 \delta & \beta \\
\beta & \beta & -\varphi
\end{array}\right| & =\beta\left|\begin{array}{cc}
2 \theta & \beta \\
-2 \delta & \beta
\end{array}\right|-\beta\left|\begin{array}{cc}
-2 \delta & \beta \\
2 \theta & \beta
\end{array}\right|-\varphi\left|\begin{array}{cc}
-2 \delta & 2 \theta \\
2 \theta & -2 \delta
\end{array}\right| \\
& =4 \theta \beta^{2}+4 \delta \beta^{2}-\left(4 \delta^{2}-4 \theta^{2}\right) \varphi \\
& =2(\delta+\theta)\left[2 \beta^{2}-2(\delta-\theta) \varphi\right] .
\end{aligned}
$$

Hence, the above proposition holds.

For the solution of $\max S$, we present the following conclusion.

Theorem 2. (33) is a sufficient condition for guaranteeing that max $S$ has a unique and reasonable solution.

Proof. All first-order partial derivatives of $S$ are

$$
\left\{\begin{array}{l}
\frac{\partial S}{\partial q_{b}}=\lambda a-2 \delta p_{b}+2 \theta p_{e}+\beta \tau, \\
\frac{\partial S}{\partial q_{e}}=(1-\lambda) a-2 \delta p_{e}+2 \theta p_{b}+\beta \tau, \\
\frac{\partial S}{\partial \tau}=\beta p_{b}+\beta p_{e}+k-\varphi \tau .
\end{array}\right.
$$


By deriving second-order derivatives of $S$, we have the corresponding Hessian matrix as follows:

$$
\left.H \prime=\left[\begin{array}{ccc}
\frac{\partial^{2} S}{\partial q_{b}^{2}} & \frac{\partial^{2} S}{\partial q_{b} \partial q_{e}} & \frac{\partial^{2} S}{\partial q_{b} \partial \tau} \\
\frac{\partial^{2} S}{\partial q_{e} \partial q_{b}} & \frac{\partial^{2} S}{\partial q_{e}^{2}} & \frac{\partial^{2} S}{\partial q_{e} \partial \tau} \\
\frac{\partial^{2} S}{\partial \tau \partial q_{b}} & \frac{\partial^{2} S}{\partial \tau \partial q_{e}} & \frac{\partial^{2} S}{\partial \tau^{2}}
\end{array}\right]=\mid \begin{array}{ccc}
-2 \delta & 2 \theta & \beta \\
2 \theta & -2 \delta & \beta \\
\beta & \beta & -\varphi
\end{array}\right] .
$$

Because $\delta>\theta>0$, by examining each order determinant of $H^{\prime}$, we find that $-2 \delta<0$ and

$$
4 \delta^{2}-4 \theta^{2}>0 \text {. }
$$

Hence, inequality (33) guarantees that $H^{\prime}$ is negative definite.

Consider the following equation set derived from (36):

$$
\left\{\begin{array}{l}
\lambda a-2 \delta p_{b}+2 \theta p_{e}+\beta \tau=0 \\
(1-\lambda) a-2 \delta p_{e}+2 \theta p_{b}+\beta \tau=0 \\
\beta p_{b}+\beta p_{e}+k-\varphi \tau=0
\end{array}\right.
$$

By dealing with (39), we obtain

$$
\left\{\begin{array}{l}
p_{b}=\frac{\lambda \delta a+(1-\lambda) \theta a+(\delta+\theta) \beta \tau}{2 \delta^{2}-2 \theta^{2}}, \\
p_{e}=\frac{(1-\lambda) \delta a+\lambda \theta a+(\delta+\theta) \beta \tau}{2 \delta^{2}-2 \theta^{2}} .
\end{array}\right.
$$

By substituting (40) into (39), the value of $\tau$ is then acquired (which we denote as $\tau^{\prime}$ ):

$$
\tau^{\prime}=\frac{\beta a+2(\delta-\theta) k}{2(\delta-\theta) \varphi-2 \beta^{2}} \text {. }
$$

By substituting (41) into (40), values of $p_{b}^{\prime}$ and $p_{e}^{\prime}$ are obtained.

Apparently, max $S$ has a unique and reasonable solution under inequality (33).

Similar to the previous section, when inequality (33) does not hold, $S(\tau)$ must be a monotonic function on the domain of definition $(0,+\infty)$, which suggests that the green degree of the product should be zero or infinity (or a given upper limit) when maximizing the total revenue of the manufacturer.

On the premise that both (11) and (33) hold, we intend to compare values of $\tau^{\prime}$ and $\tau^{*}$ (determined by (25)):

$$
\tau^{*}=\frac{2 \beta\left(2 \delta^{2}+\delta \theta\right)(\delta a+\theta a)+\left(4 \delta^{2}-2 \theta^{2}\right)^{2} k-4 \delta^{2} \theta^{2} k}{\left(4 \delta^{2}-2 \theta^{2}\right)^{2} \varphi-4 \delta^{2} \theta^{2} \varphi-4(\delta+\theta)\left(2 \delta^{2}+\delta \theta\right) \beta^{2}} .
$$

Transforming (41) as

$$
\tau^{\prime}=\frac{\beta a+2(\delta-\theta) k}{2(\delta-\theta) \varphi-2 \beta^{2}}=\frac{2 \beta\left(2 \delta^{2}+\delta \theta\right)(\delta a+\theta a)+4\left(\delta^{2}-\theta^{2}\right)\left(2 \delta^{2}+\delta \theta\right) k}{4\left(\delta^{2}-\theta^{2}\right)\left(2 \delta^{2}+\delta \theta\right) \varphi-4(\delta+\theta)\left(2 \delta^{2}+\delta \theta\right) \beta^{2}}
$$

we notice that

$$
\begin{aligned}
& \left(4 \delta^{2}-2 \theta^{2}\right)^{2} \varphi-4 \delta^{2} \theta^{2} \varphi-4\left(\delta^{2}-\theta^{2}\right)\left(2 \delta^{2}+\delta \theta\right) \varphi \\
& =\left(16 \delta^{4}-20 \delta^{2} \theta^{2}+4 \theta^{4}\right) \varphi-\left(8 \delta^{4}+4 \delta^{3} \theta-8 \delta^{2} \theta^{2}-4 \delta \theta^{3}\right) \varphi \\
& =\left(8 \delta^{4}-4 \delta^{3} \theta-12 \delta^{2} \theta^{2}+4 \delta \theta^{3}+4 \theta^{4}\right) \varphi \\
& =4\left(\delta^{2}-\theta^{2}\right)\left(2 \delta^{2}-\theta^{2}-\delta \theta\right) \\
& >0 .
\end{aligned}
$$

Hence, both the numerator and the denominator of $\tau^{*}$ are larger than the ones of $\tau^{\prime}$.

For the sake of comparison, we divide both $\tau^{\prime}$ and $\tau^{*}$ into two parts according to numerators. For the first part of each one, we have

$$
\begin{gathered}
\frac{2 \beta\left(2 \delta^{2}+\delta \theta\right)(\delta a+\theta a)}{4\left(\delta^{2}-\theta^{2}\right)\left(2 \delta^{2}+\delta \theta\right) \varphi-4(\delta+\theta)\left(2 \delta^{2}+\delta \theta\right) \beta^{2}} \\
>\frac{2 \beta\left(2 \delta^{2}+\delta \theta\right)(\delta a+\theta a)}{\left(4 \delta^{2}-2 \theta^{2}\right)^{2} \varphi-4 \delta^{2} \theta^{2} \varphi-4(\delta+\theta)\left(2 \delta^{2}+\delta \theta\right) \beta^{2}}
\end{gathered}
$$

For the second part, in order to make a comparison concisely, we denote

$$
\frac{4\left(\delta^{2}-\theta^{2}\right)\left(2 \delta^{2}+\delta \theta\right) k}{4\left(\delta^{2}-\theta^{2}\right)\left(2 \delta^{2}+\delta \theta\right) \varphi-4(\delta+\theta)\left(2 \delta^{2}+\delta \theta\right) \beta^{2}}=\frac{U k}{U \varphi-V}
$$




$$
\begin{aligned}
& \frac{\left(4 \delta^{2}-2 \theta^{2}\right)^{2} k-4 \delta^{2} \theta^{2} k}{\left(4 \delta^{2}-2 \theta^{2}\right)^{2} \varphi-4 \delta^{2} \theta^{2} \varphi-4(\delta+\theta)\left(2 \delta^{2}+\delta \theta\right) \beta^{2}} \\
& =\frac{(U+\Delta) k}{(U+\Delta) \varphi-V},
\end{aligned}
$$

where $\Delta>0$. Then,

$$
\frac{U k}{U \varphi-V}-\frac{(U+\Delta) k}{(U+\Delta) \varphi-V}=\frac{\Delta V k}{(U \varphi-V)[(U+\Delta) \varphi-V]}>0 .
$$

Given the above, $\tau^{\prime}>\tau^{*}$ under the same green degree subsidy $k$. Considering the decision model of the government,

$$
\begin{array}{ll}
\max & k \\
\text { s.t. } & \tau k \leq g,
\end{array}
$$

we draw the following conclusion:

Proposition 2. Under a finite financial budget, the per-unit green degree subsidy of the centralized supply chain provided by the government is always lower than the one of the decentralized supply chain.

Considering the inverse relation between $\tau$ and $k$, we present the following result by Proposition 2:

Corollary 1. The green degree of the product of the centralized supply chain is always higher than the one of the decentralized supply chain.

Specifically, the decision model of the government is given as follows:

$$
\begin{array}{ll}
\max & k \\
\text { s.t. } & \frac{\beta a k+2(\delta-\theta) k^{2}}{2(\delta-\theta) \varphi-2 \beta^{2}} \leq g .
\end{array}
$$

Next, we analyze the profits of the two types of supply chain. Clearly, objective functions of total profits of the two supply chains are the same:

$$
S=M+R_{b}+R_{e} .
$$

However, their sales prices must be different under quadratic profit functions [24]. Certainly, we can accurately calculate the difference between them by comparing (9) and (40).

Because a quadratic function has only one optimal solution, we have the following result:

$$
\max S>\max M+\max R_{b}+\max R_{e} .
$$

Namely, the total profit of the centralized supply chain is always higher than the one of the decentralized supply chain. In the next section, we will show this point by an example.

\section{Numerical Illustration on Brick Production}

Before illustrating the performance of the developed models, we briefly discuss the government policies in energy-saving production. Although the entity enterprises substantially contribute to the gross domestic product (GDP) in many developing countries, they often utilize outdated, energyintensive technologies to carry out industrial production activities [21], which lead to serious pollution on environment.

The promotion of green production is one important aspect to lower electricity consumption and oil consumption. Hence, the government pays much attention to strengthen the awareness of green production. In China, for example, the government provides energy-saving subsidies for many electrical and petrolic appliances. Both manufacturers and consumers benefit from this energy-saving policy.

In order to visualize the models, we present an example with the following scenario: given market potential demand $a=1000$, consumers' purchasing preference proportions for the physical store retailer and the e-commerce retailer are $\lambda=0.6$ and $1-\lambda=0.4$, the marginal demand with respect to the sales price is $\delta=3$, the demand shift with respect to the price is $\theta=2$, the marginal demand with respect to the green degree level is $\beta=1$, and the fixed cost related to the green degree is $\varphi=2$. In addition, we assume that the financial budget of the government to motivate green production is $g=125000$. Apparently, the above parameters meet both inequality (11) and inequality (33).

First, the decentralized case is analyzed. By (9), the undetermined equilibrium solution of the Bertrand game is obtained:

$$
\left\{\begin{array}{l}
p_{b}^{*}=\frac{4400+18 q_{b}+6 q_{e}+8 \tau}{32}, \\
p_{e}^{*}=\frac{3600+6 q_{b}+18 q_{e}+8 \tau}{32} .
\end{array}\right.
$$

Clearly, optimal prices of the two retailers are determined as soon as the manufacturer makes his decisions.

Examining (25) and (26), we find that optimal decisions of the manufacturer are ultimately determined by the perunit green degree subsidy. Thus, according to (31), we consider the following model:

$$
\begin{array}{ll}
\max & k \\
\text { s.t. } & \frac{240000 k+640 k^{2}}{800} \leq 125000, \quad k \geq 0 .
\end{array}
$$

We transform the inequality in the constraint to

$$
20 k^{2}+7500 k-3125000 \leq 0 .
$$

By the factorization approach, we have

$$
(4 k+2500)(5 k-1250) \leq 0 .
$$

Hence, $\max k=250$ on the interval $[0,250]$.

Given the above, the green degree of the product is determined by (25): $\tau^{*}=500$. And the optimal wholesale prices are obtained by (26): $q_{b}^{*}=510$ and $q_{e}^{*}=490$. By all the obtained results, optimal prices of the physical store retailer and the online retailer are $p_{b}^{*}=641.25$ and $p_{e}^{*}=608.72$. The 


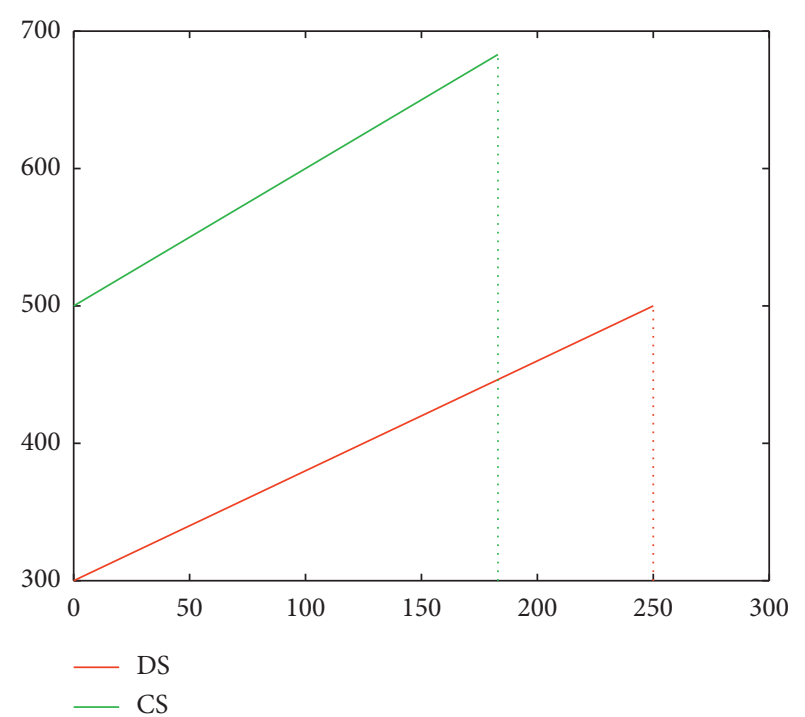

Figure 1: Curves of $\tau^{*}$ and $\tau^{\prime}$ (DS and CS, respectively) with respect to $k$.

profit of each participant is as follows: $M^{*}=250388.5$, $R_{b}^{*}=51671.8$, and $R_{e}^{*}=42304.7$. The total profit of the supply chain is $M^{*}+R_{b}^{*}+R_{e}^{*}=344365$.

Second, we handle the centralized case. According to (50), we consider the following model:

$$
\begin{array}{ll}
\max & k \\
\text { s.t. } & \frac{1000 k+2 k^{2}}{2} \leq 125000 .
\end{array}
$$

Then, we obtain $\max k=183<250$, which coincides with Proposition 2. Substituting $k=183$ into (41), we have $\tau^{\prime}=683$. Substituting $\tau^{\prime}=683$ into (40), we obtain $p_{b}^{\prime}=601.5$ and $p_{e}^{\prime}=581.5$. Finally, the profit of the centralized supply chain (or a manufacturer who operates two sales channels) is $\max S=359244.5>M^{*}+R_{b}^{*}+R_{e}^{*}$.

The curves of the two green degrees of the decentralized supply chain (DS) and the centralized supply chain (CS) are depicted in Figures 1 and 2.

It is shown that the difference between $\tau^{*}$ and $\tau^{\prime}$ is highest when $k=183$.

Next, we analyze the sensitivity of the total profit of each supply chain with respect to the coefficient of the extra production cost $\varphi$ under a certain market; i.e., $a, \lambda, \delta, \theta$, and $\beta$ remain unchanged. According to (12) and (34), the value range of $\varphi$ is $\varphi>1$. Thus, we consider $\varphi \in[1.5,5]$.

The curves are presented in Figure 2.

The curve of CS is always above the curve of DS, which meets our conclusion. Besides, with the increase of $\varphi$, the two curves get closer quickly. Hence, we draw the following conclusion: when $\varphi$ is relatively low, the centralized decision-making of the supply chain significantly raises the total profit; when $\varphi$ is relatively high, the motivation of the centralized decision-making reduces.

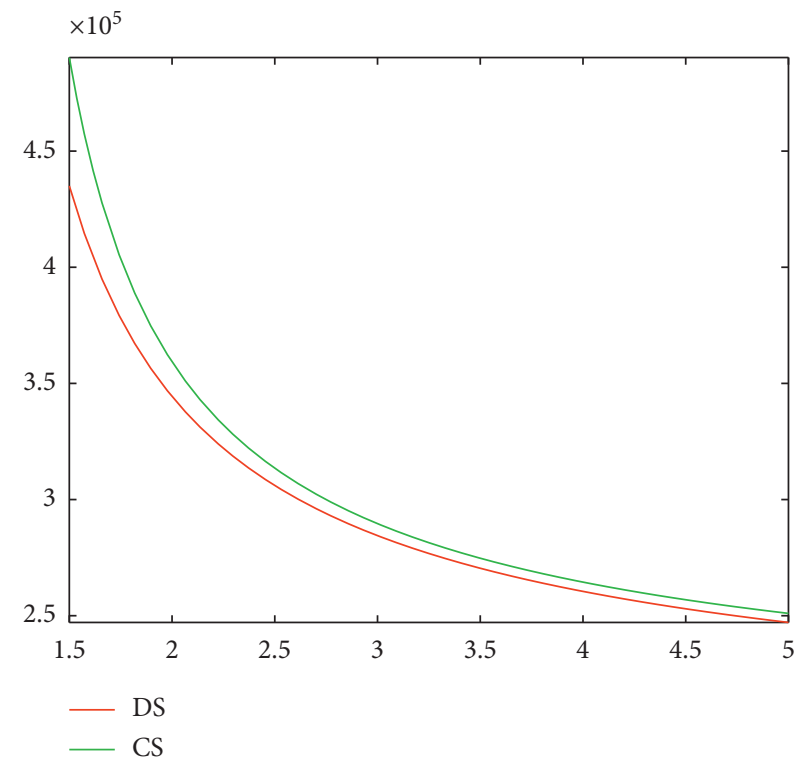

Figure 2: Curves of total profits of DS and CS with regard to $\varphi$.

\section{Conclusions}

In this paper, we investigate production and pricing issues in a two-echelon dual-channel supply chain under government subsidies. Both decentralized supply chain and centralized supply chain are considered. In the decentralized supply chain, we propose a three-layer decision model involving the government, the manufacturer, and dual-channel retailers. Both Bertrand game and Stackelberg game are conducted to deal with the multilevel model. The existence and uniqueness of the solution of each game model is analyzed. In the centralized supply chain, a two-layer model for determining sales prices, the green degree, and the per-unit green degree subsidy is constructed. Decision models of the government under a financial budget are developed for maximizing green degrees of the two cases.

This paper reveals some managerial insights. First, relations between all the coefficients are analyzed for the two cases in order to guarantee that each supply chain runs normally. Second, we demonstrate that the green degree of the product of the centralized supply chain is always higher than the one of the decentralized supply chain, which means that the government tends to offer production subsidies to a centralized supply chain. Third, it is shown that a centralized supply chain always gains more profit than a decentralized one.

Nevertheless, some aspects have not been taken into consideration. First, demand functions are only assumed to be related to sales prices. However, in practice, they may vary with time, especially for some seasonal products. In such situations, dynamic pricing strategies are often adopted. Second, competition between different supply chains have not been considered. Besides, the impact on the environment incurred by green production has not been paid 
attention in this paper. In practice, consumers' awareness on the environment attracts much attention in recent years.

\section{Data Availability}

The data used to support the findings of this study are included within the article.

\section{Conflicts of Interest}

The authors declare that there are no conflicts of interest regarding the publication of this paper.

\section{References}

[1] S. Swami and J. Shah, "Channel coordination in green supply chain management," Journal of the Operational Research Society, vol. 64, no. 3, pp. 336-351, 2013.

[2] S. Asian, A. Hafezalkotob, and J. J. John, "Sharing economy in organic food supply chains: a pathway to sustainable development," International Journal of Production Economics, vol. 218, pp. 322-338, 2019.

[3] G. Xie, W. Y. Yue, W. R. Liu, and S. Y. Wang, "Risk based selection of cleaner products in a green supply chain," Pacific Journal of Optimization, vol. 8, no. 3, pp. 473-484, 2012.

[4] G. Xie, "Modeling decision processes of a green supply chain with regulation on energy saving level," Computers \& Operations Research, vol. 54, pp. 266-273, 2015.

[5] Q. Zhang, W. Tang, and J. Zhang, "Green supply chain performance with cost learning and operational inefficiency effects," Journal of Cleaner Production, vol. 112, no. 4, pp. 3267-3284, 2016.

[6] W. Zhu and Y. He, "Green product design in supply chains under competition," European Journal of Operational Research, vol. 258, no. 1, pp. 165-180, 2017.

[7] H. H. Song and X. X. Gao, "Green supply chain game model and analysis under revenue-sharing contract," Journal of Cleaner Production, vol. 170, no. 3, pp. 183-192, 2018.

[8] Z. Lu and S. Shao, "Impacts of government subsidies on pricing and performance level choice in energy performance contracting: a two-step optimal decision model," Applied Energy, vol. 184, pp. 1176-1183, 2016.

[9] X. Zhang, X. Xu, and P. He, "New product design strategies with subsidy policies," Journal of Systems Science and Systems Engineering, vol. 21, no. 3, pp. 356-371, 2012.

[10] Y. Yu, X. Han, and G. Hu, "Optimal production for manufacturers considering consumer environmental awareness and green subsidies," International Journal of Production Economics, vol. 182, pp. 397-408, 2016.

[11] A. Hafezalkotob, "Modelling intervention policies of government in price-energy saving competition of green supply chains," Computers \& Industrial Engineering, vol. 119, pp. 247-261, 2018.

[12] S. Safarzadeh and M. Rasti-Barzoki, "A game theoretic approach for pricing policies in a duopolistic supply chain considering energy productivity, industrial rebound effect, and government policies," Energy, vol. 167, pp. 92-105, 2019.

[13] I. E. Nielsen, S. Majumder, and S. Saha, "Game-theoretic analysis to examine how government subsidy policies affect a closed-loop supply chain decision," Applied Sciences, vol. 10, no. 1, p. $145,2020$.

[14] I. E. Nielsen, S. Majumder, S. S. Sana, and S. Saha, "Comparative analysis of government incentives and game structures on single and two-period green supply chain," Journal of Cleaner Production, vol. 235, pp. 1371-1398, 2020.

[15] W. K. Chiang, D. Chhajed, and J. D. Hess, "Direct marketing, indirect profits: a strategic analysis of dual-channel supplychain design," Management Science, vol. 49, no. 1, pp. 123$142,2003$.

[16] J. Chen, H. Zhang, and Y. Sun, "Implementing coordination contracts in a manufacturer stackelberg dual-channel supply chain," Omega, vol. 40, no. 5, pp. 571-583, 2012.

[17] B. Li, M. Zhu, Y. Jiang, and Z. Li, "Pricing policies of a competitive dual-channel green supply chain," Journal of Cleaner Production, vol. 112, no. 3, pp. 2029-2042, 2016.

[18] T. Javadi, N. Alizadeh-Basban, S. Asian, and A. Hafezalkotob, "Pricing policies in a dual-channel supply chain considering flexible return and energy-saving regulations," Computers \& Industrial Engineering, vol. 135, pp. 655-674, 2019.

[19] P. Liu and S.-P. Yi, "Pricing policies of green supply chain considering targeted advertising and product green degree in the big data environment," Journal of Cleaner Production, vol. 164, pp. 1614-1622, 2017.

[20] S. Saha, S. Majumder, and I. E. Nielsen, "Is it a strategic move to subsidized consumers instead of the manufacturer?" IEEE Access, vol. 7, pp. 169807-169824, 2019.

[21] A. Hafezalkotob, "Competition, cooperation, and coopetition of green supply chains under regulations on energy saving levels," Transportation Research Part E: Logistics and Transportation Review, vol. 97, pp. 228-250, 2017.

[22] D. Ghosh and J. Shah, "A comparative analysis of greening policies across supply chain structures," International Journal of Production Economics, vol. 135, no. 2, pp. 568-583, 2012.

[23] N. Amrouche and R. Yan, "A manufacturer distribution issue: how to manage an online and a traditional retailer," Annals of Operations Research, vol. 244, no. 2, pp. 257-294, 2016.

[24] Z. K. Lou, F. J. Hou, and X. M. Lou, "Optimal ordering and pricing models of a two-echelon supply chain under multiple times ordering," Journal of Industrial and Management Optimization, pp. 1-13, 2020. 